\title{
A Beam Shape Oscillation Monitor for HERA
}

\author{
O.V. Afanasyev ${ }^{1}$, A.B. Baluev ${ }^{1}$, K.I. Gubrienko ${ }^{1}$, E.A. Merker ${ }^{1}$, \\ K. Wittenburg ${ }^{2}$, I. Krouptchenkow ${ }^{2}$ \\ ${ }^{1}$ IHEP, Protvino, 142281, Russia \\ ${ }^{2}$ DESY, Hamburg, 22607, Germany
}

\begin{abstract}
The perfect matching of the injecting beam phase space with the accelerator lattice is a very important problem. Its successful solution allows excluding possible mismatch emittance blow-up and worsening of the beam characteristics, that is necessary to get the highest possible luminosity in hadron accelerators. The mismatch can be controlled by measuring sizes oscillation on the first revolutions of the injected beam at a certain orbit point. Designed for this purpose the construction, acquisition electronics, software controlling of the operation and data processing of such a monitor are described. A first test result with beam is presented.
\end{abstract}

Keywords: Emittance, beam profile, OTR.

PACS: 29.27.Fh, 29.27.Ac, 29.20.Dh

\section{INTRODUCTION}

The magnet structure of a circular accelerator is strong periodically, that defines the same periodicity of the Twiss parameters, describing the changing of the orientation and shape of the machine transverse phase space ellipse along the orbit. If the transverse phase space ellipse occupied by the particles of the injected beam has the same center position, shape and orientation as that one of the machine in the injection point, they will be preserved on each following turn. If this is not the case there will be an oscillation of the position and modulation of the beam dimension from turn to turn, that leads after filamentation to a blow-up of the beam emittance. The depth of the modulation depends on the degree of the mismatch between the two phase spaces and its frequency is defined by the fractional part of the tune.

The position offset can be easily defined and compensated using the usually existing beam position measurement and correction systems. But the detecting and compensation of the mismatch of transverse phase space ellipses is more complicated because the traditional profile measurement methods in proton colliders are based on methods with integration times of more than one turn and/or inserting an essentially number of matter in the beam.

An attractive method is proposed and described in [1], in which a beam shape oscillation monitor based on registration of the optical transition radiation (OTR), generated on the boundary of a metallic foil inserted in the beam is used. The theory and experimental investigation of OTR are described in many publications (see for example [2] and references there).Using OTR as a signal source of the beam dimension in our case is preferable because of some of its special properties: One needs to insert in the beam only a few micrometers thick metal foil. That allows to 
reduce considerably the influence of the beam emittance blow-up due to Coulomb scattering and ionization losses. This also considerably decreases the level of the radiation background and activation of the equipment. And last but not least a thin titanium foil will not interfere seriously with the superconducting part of the machine, even in case of a breakdown. The OTR is concentrated in a cone having a characteristic semi-vertical angle of $\sim \gamma^{-1}$ ( $\gamma$ is the relative particle energy). If the foil inclination is $45^{\circ}$, the cone axis forms an angle of $90^{\circ}$ relative to the particle trace that makes the observation of the radiation easy. The electrical noises are essentially reduced at the signal transmission because the funneling of the radiation to the sensor is done through a glass window and there are no direct electrical contacts between the transmission line and the main accelerator equipment in the tunnel.

\section{CONSTRUCTION OF THE MONITOR.}

The described beam oscillations monitor is intended for the controlling of the

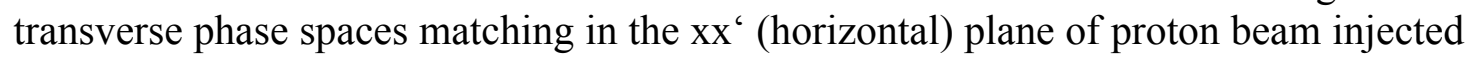
from PETRA into HERA. The proton energy at the injection is $40 \mathrm{GeV}$, the intensity for these measurements were restricted to less than $5^{*} 10^{11}$ protons/pulse and to less than 100 beam crossings to save the OTR screen. The revolution time of HERA is about $21 \mu \mathrm{s}$.

The monitor is installed in the HERA tunnel in a specially manufactured vacuum box of a cross-like form (fig.1). On the surface were denoted special geodesic marks for the precise alignment. The main functional parts of the monitor are:

- A transition radiation radiator (TRR), moved by a pneumatic driver,

- an optical objective matching the beam image with the sensitive area of the light detector,

- a module of the acquisition electronics, on which the detector is fixed.

These parts and the power supply of the electronics module are disposed in the accelerator tunnel. A computer (PC) that controls the Monitor operation and processes the information from the acquisition electronics is installed in an equipment room about $50 \mathrm{~m}$ away from the monitor.

The TRR is a 10 microns thick titanium foil, stretched on an aluminum frame, having a ring shape. To exclude the beam interaction with the frame its inner diameter is larger than the dimension of the injecting beam. To reduce the beam blow-up and to limit uncontrolled losses, the maximum number of the foil crossing is restricted to 100 beam crossings (turns), after which the beam is automatically ejected by a special designed injection trigger delay. The influence of the Coulomb scattering and the energy losses on the measurement results can be easily taken into account using the very well known relations (see e.g.[1]).

We had chosen a sensor from Dalsa, a charge-coupled device (CCD) IL-C6B performed as a line structure having 2048 pixels of the dimension $13 \times 500 \mu \mathrm{m}^{2}$. It provides a sensitivity of $\sim 115 \mathrm{~V} / \mu \mathrm{J} / \mathrm{cm}^{2}$ at a wavelength of $750 \mathrm{~nm}$. The sensitive range stretches from $\sim 550$ to $870 \mathrm{~nm}(>70 \%)$. The available frequency of the output data is $15 \mathrm{MHz}$. We had chosen this line type sensor, on one hand, to obtain data within one beam revolution period and, on the other hand, to have enough sensitivity for the light sampling. To satisfy these requirements we have developed a control and 
acquisition electronics, which need about 100 ns to process the information of one pixel. It means that during $20 \mu \mathrm{s}(1 \mu \mathrm{s}$ less than the revolution period, necessary for proper timing and readout) we can treat not more than 200 pixels. This has two consequences: 1) the transition radiation spot should not occupy more than 200 last pixels along the sensor, 2) a pretrigger of about $300 \mu$ s before the beam injection and measurement start is necessary to reset all 2048 photocells and clean the shift register.

Calculations in $[3,4,5]$ show, that a charged particle of some dozens of $\mathrm{GeV}$ energy, crossing a metallic surface, emits in back direction nearly $10^{-2}-10^{-3}$ photons/particle in the optical range. Certainly this figure essentially depends on the electromagnetic properties of the foil. We couldn't find any experimental data of these characteristics for $\mathrm{Ti}$ in the visible range. To calculate the amount of photons $\mathrm{N}_{\mathrm{p}}$ receiving the sensor, we assume:

- the pessimistically variant of the radiation efficiency $\left(10^{-3}\right.$ photons/proton),

- that the beam (and consequently the radiation on the sensor) has a Gaussian distribution

- that the radiation occupies all 200 pixels accessible for the registration $( \pm 3$ $\sigma, 1 \sigma \approx 33$ pixels).

Then, in the maximum of the distribution, we get:

$\mathrm{N}_{\mathrm{p}}=5 \cdot 10^{11} \cdot 10^{-3} /(2 \pi)^{-1 / 2} \cdot 33=0.6 \cdot 10^{7}$ photon $/$ pixel.

At the maximum of the sensitivity (wavelength $750 \mathrm{~nm}$ ) this corresponds to an energy $\mathrm{E}$ of

$$
\mathrm{E}=3 \cdot 10^{8} \cdot 6.6 \cdot 10^{-34} \cdot 0.6 \cdot 10^{7} / 750 \cdot 10^{-9}=1.6 \cdot 10^{-12} \mathrm{~J} / \text { pixel. }
$$

Here $3 \cdot 10^{8} \mathrm{~m} / \mathrm{sec}$ is the light speed and $6.6 \cdot 10^{-34}$ the Planck's constant. Taking into account the sensitivity we can get an output voltage $\mathrm{V}$ of:

$$
\mathrm{V}=115 \cdot 1.6 \cdot 10^{-12} / 0.05 \cdot 14 \cdot 10^{-4} \cdot 10^{-6}=2.6 \mathrm{~V}
$$

This voltage is sufficient for the normal processing.

The SSD line is fixed in a slot in the front panel of the electronics module. The information received from the output of the sensor is digitized, stored in a local memory and transferred to the PC after finishing the measurement in each accelerator cycle.

The necessary dimension of the light spot on the sensor is provided by the optical objective consisting of two cylindrical lenses (to provide independent demagnification for each direction) and a common spherical lens for both directions. The objective is mounted in a way, that in the image plane the centre of TRR coincides with the front focus while the sensor is put in the back one. This arrangement ensures a transformation of an image point to a point on the sensor. The relation of the beam dimension to the spot dimension on the sensor will be equal to the relation of the back focus length of the objective to the length of front one. In our case it is 20:104. In the vertical plane the objective parameters are chosen to get a minimum possible spot dimension, to focus the maximum amount of light. We got a transforming coefficient 
of 20:310. If the beam diameter, as we suppose, is about $10 \mathrm{~mm}$, we have a spot of about $1.9 * 0.6 \mathrm{~mm}^{2}$ on the sensor. Therefore we expected a signal dimension of $\sim 136$ pixels along the line (horizontal) and a little bit more than the pixel size in the vertical plane. Therefore the positioning of the sensor in the vertical plane is critical and was done very carefully.

The objective, the electronic module and the pneumatic driver are installed on a common platform screwed to the box having special seating places for this. Such an arrangement on one common platform allows a precise adjustment between them and their position in respect to the box axis. Also additional alignments are foreseen of the electronics module and consequently of the CCD-line in regards to the objective axis: a manual adjustment is provided along the line and a remote one is provided by a stepper motor in the transverse direction. To conserve the high vacuum condition the transmission of the movement of the TRR in and from the beam is done through a membrane bellow by a pneumatic driver.

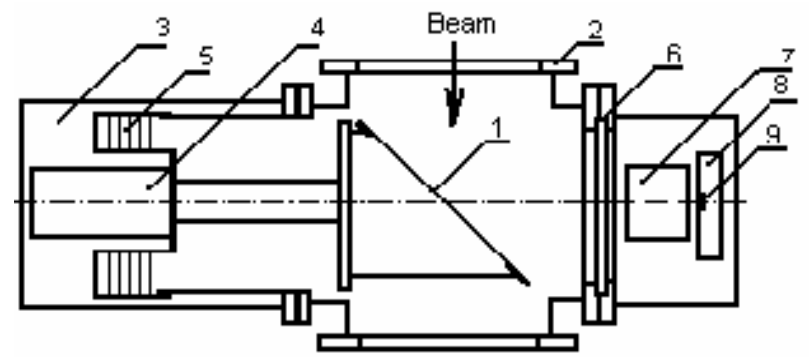

FIGURE 1. Sketch of the Monitor construction: 1-RR, 2-Box, 3-Platform, 4-Pneumatic driver, 5Membrane bellow, 6-Glas window, 7- Objective, 8-Electonic 9-CCD-line.

\section{THE TESTING AND COMMISSION OF THE MONITOR.}

The most delicate problem during the Monitor commission was the alignment of the TRR coordinate and its inclination to get an angle of $45^{\circ}$ between its surface and both the box and the objective axes. The problem was solved in some consecutive steps. First of all the box and a diode laser were fixed on a special support. Using the laser and prepared auxiliary equipment we found the box axes. After that the module support was adjusted that the box axis and the laser ray came precisely in the horizontal plane. Then the TRR was fixed that the laser ray crossed it in the geometrical centre. In the next step we achieve the reflected ray being precisely directed along a vertical by the aligning of the TRR. The objective was fastened to the common platform in such a manner that the reflections from the TRR laser ray don't change its position and direction. Finally the position of the electronics module and the CCD sensor were adjusted by controlling the location of the laser spot on the sensor and by maximizing the signal level. The orientation of the box is carefully measured using the geodesic marks on the box and had taken into account during the mounting of the box in the tunnel. For the alignment we used a Lasris Diode laser having continued and modulation modes of operation. The last one is very useful for the testing and tuning of the acquisition electronics and general timing. After the mounting of the Monitor in the HERA tunnel it was necessary to include the monitor 
in the common accelerator control and timing systems: It had to be guaranteed that the movement of the TRR happens only without a beam in the accelerator. For the correct measurement a pretrigger of $\sim 300 \mu$ s before the beam injection was prepared from the general accelerator timing system and delivered to the electronics module for basing the photocells. After the injection a series of precise revolution triggers were necessary to execute the data sampling. For insuring the coincidence of the first TRR beam crossing and of the start of the measurement an additional signal generated by a photomultiplier-scintillation-counter (PMT) located near the Monitor was used. The PMT was sensitive to beam losses during the first pass of the beam.

A beam profile measured at the first revolution is presented on Fig.2. The width at half maximum is nearly $4 \mathrm{~mm}$ for about $5 \cdot 10^{11}$ protons in 10 successive bunches. One can see some remarkable asymmetry of the distribution which will be studied more in the future. Meanwhile, during the last year of operation the injected beam emittance of HERA has already shrunk down to less than the design value due to further improvements of the per-accelerator chain of HERA. Therefore the necessity for measurements in the multiturn regime had decreased and they are still postponed.

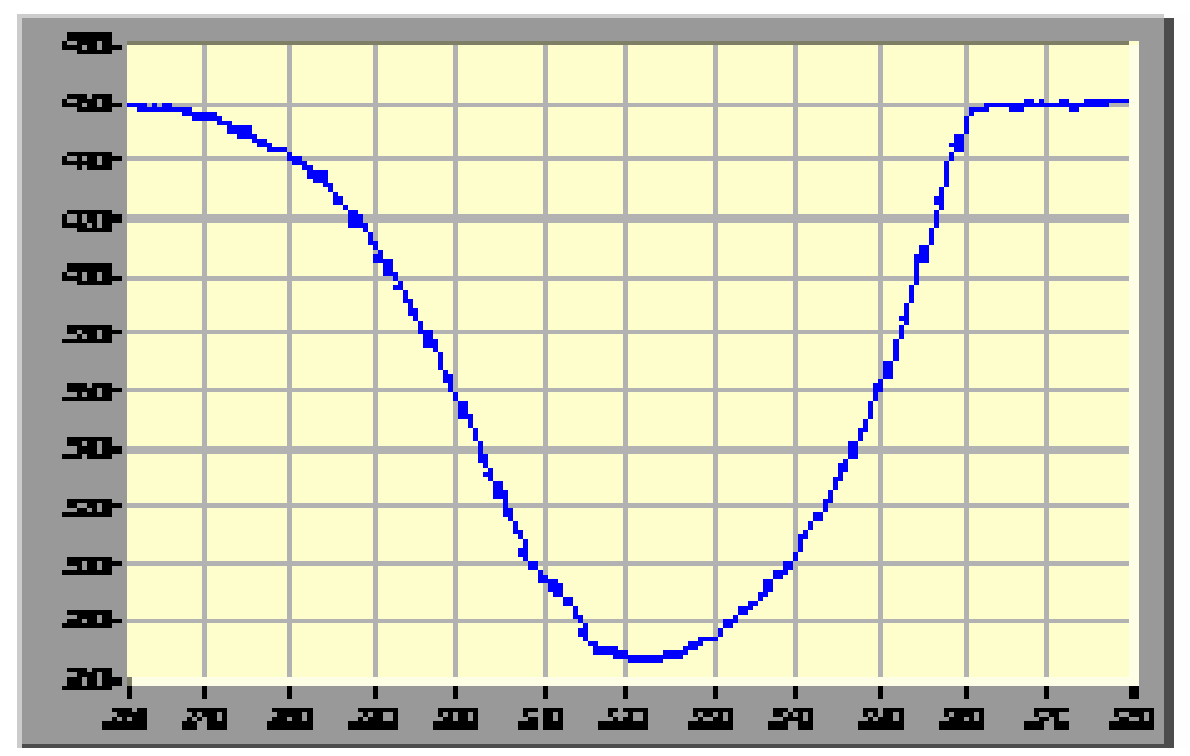

FIGURE 2. On ordinate the beam distribution in relative units in $\mathrm{x}$ direction is indicated. On abscissa the pixels numbers are displayed. 


\section{ACKNOWLEDGMENTS}

We like to thank the HERA shift crew for preparing nice injections for our measurements. The help of P. Schmid for providing us the dump trigger and H.T. Duhme for the pretrigger is greatly acknowledged.

\section{REFERENCES}

1. C. Bovet and R. Jung, LHC Project Report 3, Geneva, 1996

2. P. Catravas et al. Proc. of PAC, 2111, New York, (1999)

3. L. Wartski, J. Marcou and S. Roland, IEEE Trans. Nucl. Sci. NS-20 (1973)

4. J. Bosser et.al., NIM in Phys.Res. A238, 45, (1985)

5. V.L. Ginzburg and V.N. Tsytovitch, Transition radiation and Transition scattering (in russia), Moscow, 1984 